\title{
Compact 1x2 MEMS optical switches with low-actuation voltage
}

\author{
Hsin-Ta Hsieh and Guo-Dung John Su \\ Graduate Institute of Electro-Optical Engineering, National Taiwan University \\ 1, Roosevelt Road, Section 4, Taipei, Taiwan \\ Tel: +886-2-3366-3652Ｆax: +886-2-2367-7467 E-mail:gdjsu@cc.ee.ntu.edu.tw
}

\begin{abstract}
In this paper, we report a compact $1 \times 2$ MEMS optical switch actuated by less than one voltage. Over past few years, micro-electro-mechanical systems (MEMS) have emerged as a leading candidate for achieving true all-optical multiwavelength network. Due to the inheritance of mechanical vibration in the under damping condition, most MEMS switches might need external control circuit or mechanical stoppers to suppress the undesired jittering of optical signal. To overcome this difficulty, we redesigned a MEMS optical switch monolithically integrated with a vertical micromirror with large surface area. The micromirror is made by the low-cost anisotropic wet etching technique. The size of the micromirror is $500 \mu \mathrm{m} \times 1200 \mu \mathrm{m}$, which is large enough to accommodate the optical beam diameter of 62 $\mu \mathrm{m}$ from a ball lens fiber. Because the moving direction of the MEMS optical switch is perpendicular to the surface normal of the micromirror, the effect of the mechanical vibration is compensated by the large surface area of the micromirror. In addition, a capacitor of $100 \mu \mathrm{F}$ is connected in parallel with the MEMS switch to serve as a low pass filter. The switching time can be improved in the $5 \mathrm{~ms}$ range. Finally, a calculated driving waveform derived from the square wave is used as the triggering signal, after analyzing the switching performance by Fourier transform function. Our experimental results show the rising time and fall time of the MEMS switch is $3 \mathrm{~ms}$ and $3.1 \mathrm{~ms}$, respectively. The optical insertion loss caused by the micromirror is $0.45 \mathrm{~dB}$. The actuation voltage is around 0.3 volt and the switch is actuated by electromagnetic force. The footprint of the packaged device is $18 \mathrm{~mm}$ x $12 \mathrm{~mm}$. A low cost, small size and high performance optical switch is experimentally demonstrated.
\end{abstract}

Keywords: Optical MEMS, Photonic switches, Electromagnetic Actuation, Low Voltage

\section{INTRODUCTION}

Due to rapidly growth of data communication, the bandwidth of copper line is not enough. Therefore, optical fiber is an indispensable media for future communication. Optical switch is the important component for the all-optical network. E-O-E is a traditionally solution, but the E-O conversion has some intrinsic limits. First, the conversion and regeneration speed can not follow at very high speed communication. Second, high speed conversion equipment is relatively expensive, high power consumption and large size. On the other hand, all optical switching mechanism (O-O-O) does not need those conversions. A good example is the MEMS optical switches. It is low cost and small in size. It is reliable by smart mechanical design [1]. Unfortunately, inherent vibration is almost unavoidable in mechanical structure [2 5], and the mechanical displacement is amplified at resonant frequency [6]. An extra stopper is needed for landing movable structures [7]. But stopper type switches may suffer stiction problem after a long time usage. To eliminate the potential stiction problem, we adopt a stiction-free type switch and actuated by the electromagnetic force [8]. MEMS structure actuated by electromagnetic force has long travel distance. Unfortunately, it is easy to resonant without any mechanical energy absorber. Therefore, external driving circuit is required to reduce vibration. The external driving circuit can be as simple as a capacitor to cut off un-desired high frequency components or a ROM that stores a special waveform. The special waveform is released when the actuation signal is activated. All additional electric components consume power and increase package volume. The special waveform may take a lot of time to find a suitable one.

In this paper, we design a proper optical structure and try to simplify the driven circuit. Our mirror is vertical and the height is $500 \mu \mathrm{m}$. Truncation ration is more than 2 compare with $62 \mu \mathrm{m}$ beam size. Full Gaussian-like beam is reflection almost. The mirror vibration at resonant frequency is less significant for small beam size. We do the theoretical analysis

Photonics: Design, Technology, and Packaging II, edited by Derek Abbott, Yuri S. Kivshar, Halina H. Rubinsztein-Dunlop, Shanhui Fan, Proc. of SPIE Vol. 6038, 60381W, (2006) · 0277-786X/06/\$15 · doi: 10.1117/12.638426 
and improve the switching performance. First, we start with the $200 \mu \mathrm{m}$ beam diameter in the optical switch and measure its performance by square wave driving waveform. Then we reduce the beam diameter to $62 \mu \mathrm{m}$ to minimize the vibration effect. Then a capacitor is connected in parallel with the MEMS chip to cut high frequency off through the optical switch under square wave driving waveform. We analyze the optical signal in frequency domain by Fourier transfer. Based on the analysis results, a special waveform is edited by software to avoid resonant frequency component. In section two, we will discuss the switch fabrication and package design. The experimental results and discussions are shown in section three. Finally we will conclude the paper in the summary.

\section{SWITCHES FABRICATION AND PACKAGE DESIGNS}

The MEMS switch chip is a vertical mirror monolithically integrated on an actuation flap. The metal coils are patterned on the actuation flap to generate electromagnetic force with permanent magnets, which are attached with the MEMS chip. To fabricate smooth and vertical mirror, we use the silicon-on-insulator wafer with (110) substrate bonded with (100) silicon layer, as shown in Fig. 1(a). Anisotropic wet etching of TMAH (tetra-methyl ammonium hydroxide) is performed on the (110) wafer to form the vertical mirror on the (111) surface planes due to the crystal orientation. The mirror surface is smooth and stress free. Then we pattern metal coil on the top side of the (100) wafer by E-beam evaporator. The actuation flap and springs are patterned in the last step by DRIE in Fig. 4(d). The gold is deposited on the micromirror to reflect the infra red light at the wavelength of $1550 \mathrm{~nm}$ for optical communication applications. The moving direction of the actuation flap is 90 degree to the wafer surface. By doing this, the surface normal of the micromirror is perpendicular to the moving direction of the actuation flap, which has the advantage of digital switching function. Besides, the height of the micromirror is pre-determined by the thickness of the (110) wafer to accommodate optical beam with the diameter of few hundred microns. The height of the micromirror is $500 \mu \mathrm{m}$ with a length of 1200 $\mu \mathrm{m}$. The thickness of the micromirror is controlled by the etching time in the TMAH solution and is measured to be around $20 \mu \mathrm{m}$. The MEMS switching chips are very robust in terms of mechanical vibration and high G shock. Our design and experimental results showed that the MEMS switch can pass the rigorous Telcordia ${ }^{\circledR}$ GR-1221 requirements, which involve $20 \mathrm{G}$ high frequency vibrations ( from $20 \mathrm{~Hz}$ to $2000 \mathrm{~Hz}$ ) and 500G [1].

Figure 2 shows the photo picture and the schematic drawing of the compact $1 \times 2$ switch package. The switch has three ports. One pair of fibers is separated by a distance alone the same line, and the other one is on the reflection path. There is a ball lens glued to the front end of the fiber to collimate the diverging light from the fiber. The micromirror is at the intersections of the light paths from the three fibers. A pair of permanent magnets is attached to the bottom of the MEMS chip to provide necessary magnetic field. The vertical mirror is actuated by electromagnetic force from the actuation flap with two electrical contacts for current flows. When the voltage is applied, the current pass through the wire loops on the flap and an upward magnetic dipole is generated in the $\mathrm{Z}$ direction, as shown in Fig. 3(a). The magnetic field is provided by permanent magnet composed of $\mathrm{NdFeB}$ (Neodymium Iron Boron). The qusi-uniform

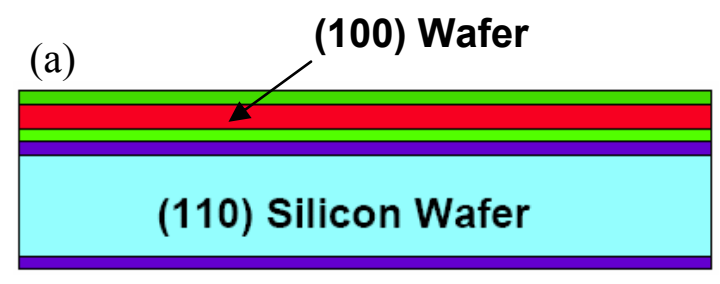

(b)

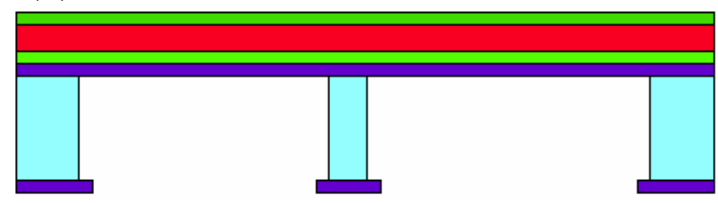

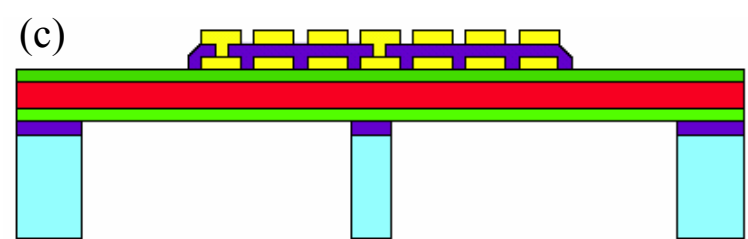

(d)

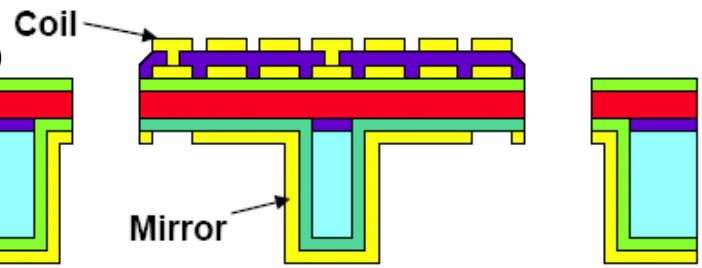

Figure 1. Fabrication processes of optical MEMS switches include (a) bond (110) and (100) wafer, (b) wet etching of the vertical mirror, (c) pattern the metal coils, and (d) pattern the actuation flap and spring. 

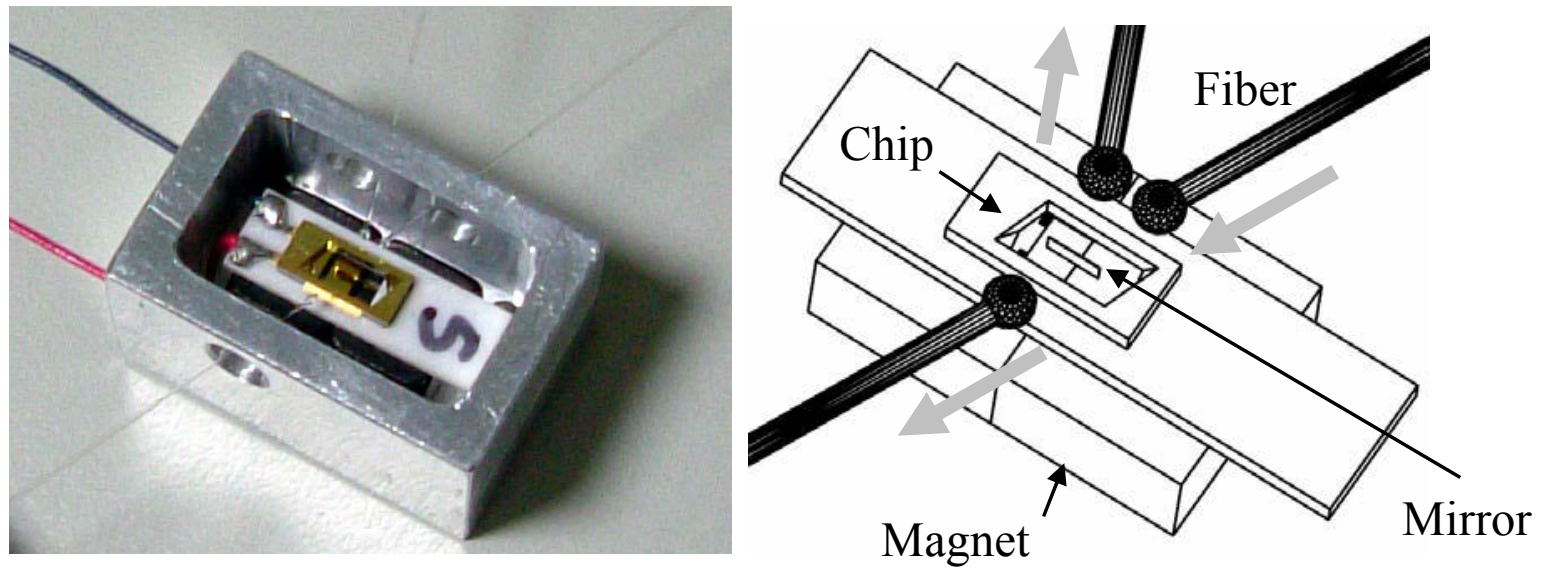

Figure 2. Picture and schematic drawing of a fully packaged 1x2 MEMS optical switch, the optical signal enters from input port can be transmitted or reflected to the output port.

magnetic field is present at the minus Y-direction on the magnet surface. The actuation flap is about $0.5 \mathrm{~mm}$ above to the magnet surface and a torque rotate along $\mathrm{X}$-axis is generated. The mirror is up and down by turning on and off the applied voltage, as shown in Fig. 3(b) and 3(c), respectively. When the voltage is on, the switch is at cross state. The light from the input port is reflected to another output fiber. When applied the voltage is removed, the mirror is down and the light from the input port pass through to another fiber directly, which is bar state. There is no power consumption at bar state.

To reduce the total package size, we decide to use the ball lens fiber to collimate the light from the single mode fiber. The size of the ball lens is around $450 \mu \mathrm{m}$ in diameter and is quite suitable for this purpose. Each magnet is $5 \mathrm{~mm} \mathrm{x}$ $6 \mathrm{~mm} \times 3 \mathrm{~mm}$, and one pair of magnets can form sufficient magnet field along the minus Y direction. The MEMS chip is diced at the size of $2 \mathrm{~mm} \times 6 \mathrm{~mm}$, which is glued on to a ceramic board for easy handling. The length, width and height of the packaged $1 \times 2$ MEMS switch is $18 \mathrm{~mm} \times 12 \mathrm{~mm} \times 10 \mathrm{~mm}$.

\section{EXPERIMENTIAL RESULTS AND DISCUSSIONS}

It is our goal to shrink the overall package size as small as possible. One of the challenges is to eliminate complicated driving circuits, while maintaining fast switching speed. There is no mechanical stopper on the MEMS chip to absorb the moving energy of the actuation flap because of the potential stiction issue. As a result, we need to carefully consider the optical design of the light path and the electrical actuation signal to avoid the MEMS structures vibrating under the resonant frequency. It is generally accepted that the switching time of a MEMS optical switch must fell in the range of few milliseconds to replace the current opto-mechanical switches. In the following paragraph, we will show our approaches to achieve this goal.

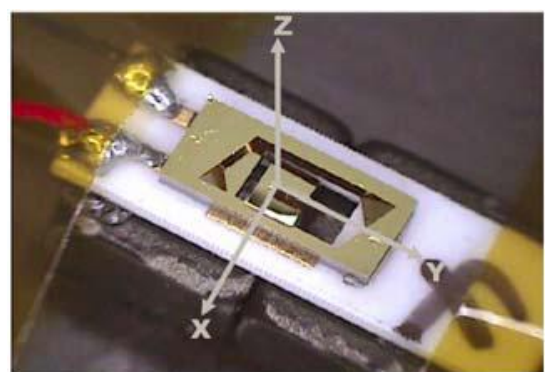

Figure 3a: the mirror on the flap is actuated by electromagnetic force.

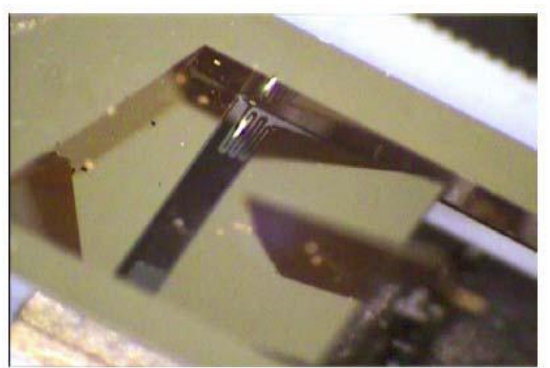

Figure 3b: the mirror is up when the voltage is on.

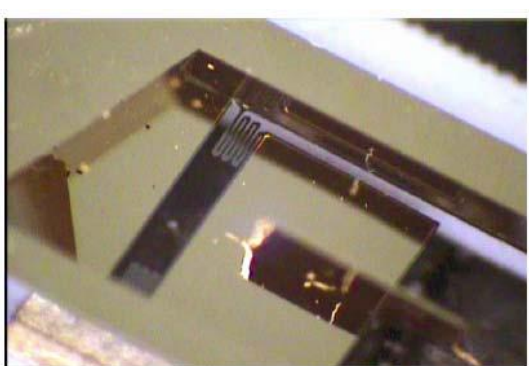

Figure 3c: the mirror is down when the voltage is off. 


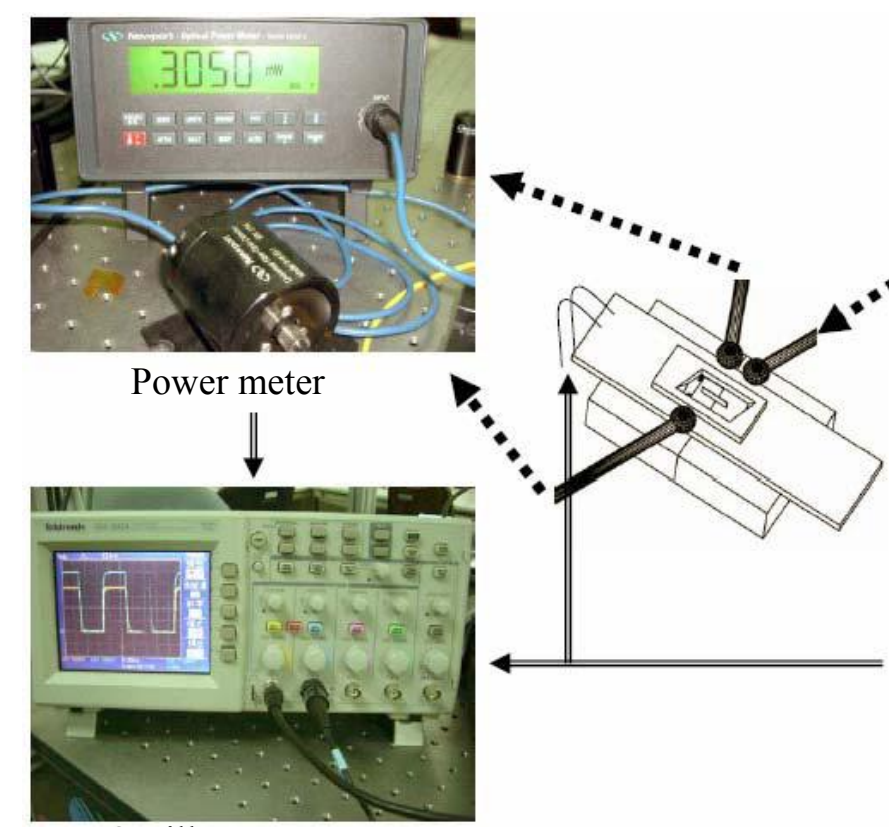

Oscilloscope

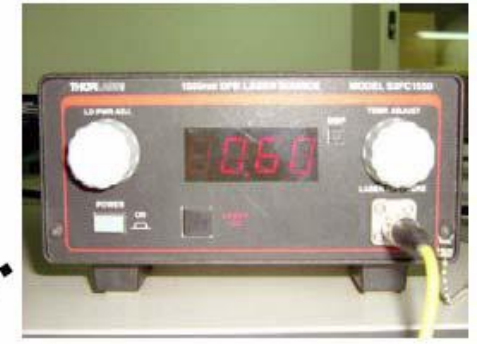

Laser source

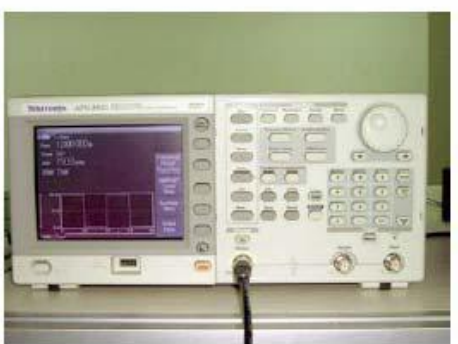

Function generator

Figure 4. Experimental setup to measure the switching time of MEMS device.

Figure 4 shows the experimental setup to measure the switching speed of the MEMS optical switch. The infra red light source is from the semiconductor laser diode at the wavelength of $1550 \mathrm{~nm}$. The output ports are connected to the power meter, which has a BNC cable output to the oscilloscope. The function generator provides a square wave to drive the switch on and off. The driving signal from the function generator is also sampled by the oscilloscope for comparison. The switching time and waveform of both optical and driving signal are shown on the screen of oscilloscope. We save this data for further analysis on the computer. The minimum resolution of the oscilloscope is $10^{-6}$ second and is sufficient for our need because the switching speed of MEMS devices at this size is typically around mille-second $\left(10^{-3}\right)$.

The light paths from the single mode fiber are parallel to the substrate surface. Since the micro mirrors are etched vertically in the fabrication process, the control mechanism of our design is traditionally classified as digital switching. The surface normal of the micromirror is kept constant during the movement of the micromirror. In other words, there is almost no angular misalignment from the micromirror itself. The most optical loss will be mainly due to the truncation ration of the micro mirror to the beam sizes. The smaller the beam diameters, the loser the alignment can be because most of the optical power can be reflected back to the output fiber by the micro mirror with large surface areas.

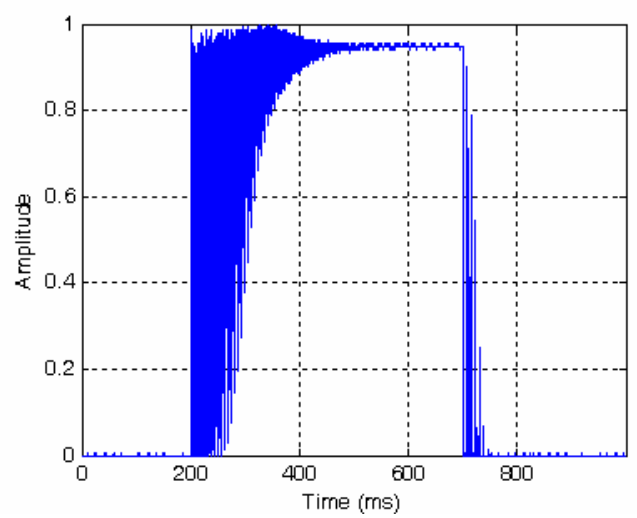

(a)

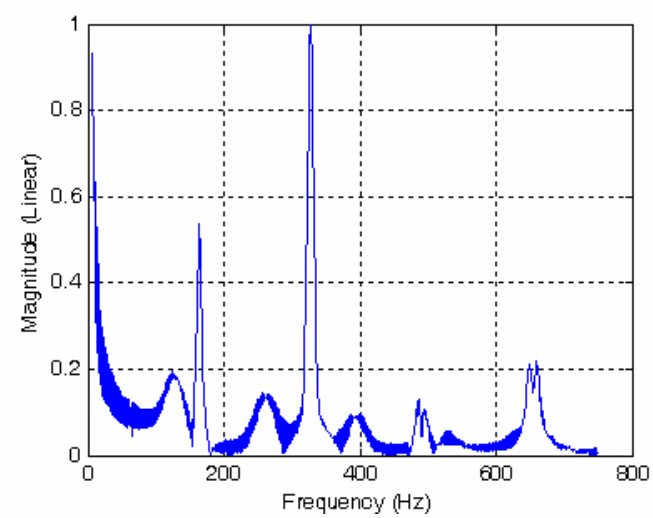

(b)

Figure 5. Switching performance from $200 \mu \mathrm{m}$ beam waist 


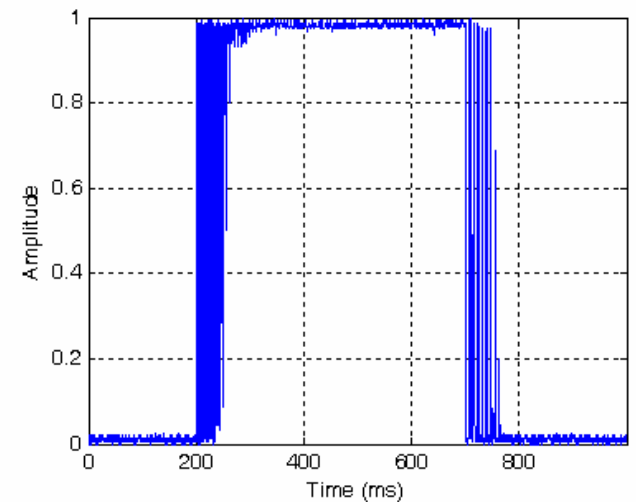

(a)

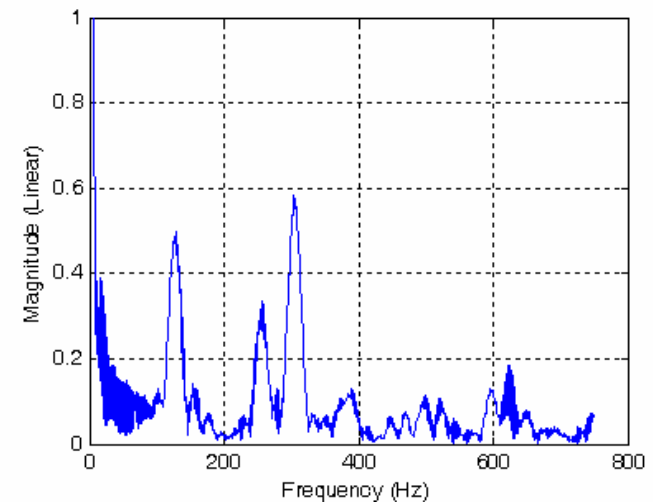

(b)

Figure 6. Switching performance from $62 \mu \mathrm{m}$ beam waist

We start with the optical beam with $200 \mu \mathrm{m}$ in diameter, which can be made by the commercial available GRIN lens of $1 \mathrm{~mm}$ focal length. The micromirror is $500 \mu \mathrm{m}$ to make the truncation ration is around 2. Large truncation ratio ensures the full laser beam is reflected. Without mechanical stopper, the mirror is expected to vibrate for few milliseconds before it is damped. If the amplitude of the micromirror vibration is too large, the laser beam may not be fully covered by the mirror and some light will pass thought it. Fig. 5(a) illustrates the optical signal waveform, which shows the switching time of about $250 \mathrm{~ms}$. This problem may be overcome by reducing the laser beam size. We use ball lens fiber made by Corning. A ball lens of $450 \mathrm{~mm}$ diameter is attached to the front end of a single mode fiber. The beam waist is only $62 \mu \mathrm{m}$. Fig. 6 (a) shows the switching time is reduced to approximately $150 \mathrm{~ms}$.

To study this effect, we take a Fourier transfer function of the Fig. 5(a) and Fig 6(a), respectively, by using the equation 1 shown below. We assume the best output optical signal is also square wave. A square wave can be made by the superposition of a series of basis, such as cosine function with different frequency and magnitude. After the transfer function, the optical signal becomes a series of frequencies which is separate and clean to see. Our signal is discrete value due to the sampling of oscilloscope. Discrete Fourier transform is needed. The calculation is done by Matlab.

$$
\begin{aligned}
& F(\omega)=\int_{-\infty}^{\infty} f(t) e^{-i \omega t} d t \\
& f(t)=\frac{1}{2 \pi} \int_{-\infty}^{\infty} F(\omega) e^{i \omega t} d \omega
\end{aligned}
$$

This long switching time is mainly due to the vibration of the MEMS switch after it is activated by the square wave and we can clearly see the resonant frequency of the MEMS switch is around $120 \mathrm{~Hz}$ and $300 \mathrm{~Hz}$ regardless the optical beam size. The mirror vibrates in large amplitude at resonant frequency. Some light are out of mirror range and the energy is lost to cause the jittering in the oscilloscope.

In order to eliminate the driving signals that may cause the MEMS mirror to vibrate in the original square wave, we can simply design a capacitor in parallel connected with MEMS chip to server as a low-pass filter so that the high frequency components in the square wave can not go through the MEMS chip, as in Fig. 7. It is evident that the dramatically changing optical signal becomes smoother by add a 100uF capacitor parallel with switch chip, as shown in Fig. 8(a). Fig. 7(b) shows the frequency spectrum of the optical signal in Fig. 8(a). The resonant peak is quite small, but not zero, compared to the previous setups (Fig. 5(b) and Fig. 6(b)). An additional capacitor is a simple low pass filter to reduce

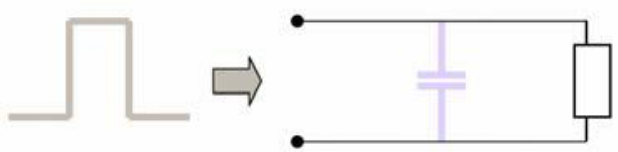

Figure 7. A $100 \mu \mathrm{F}$ capacitor parallel with switch chip 


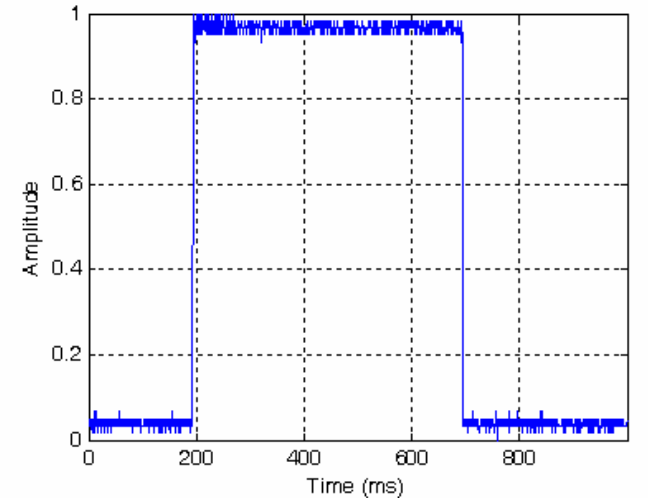

(a)

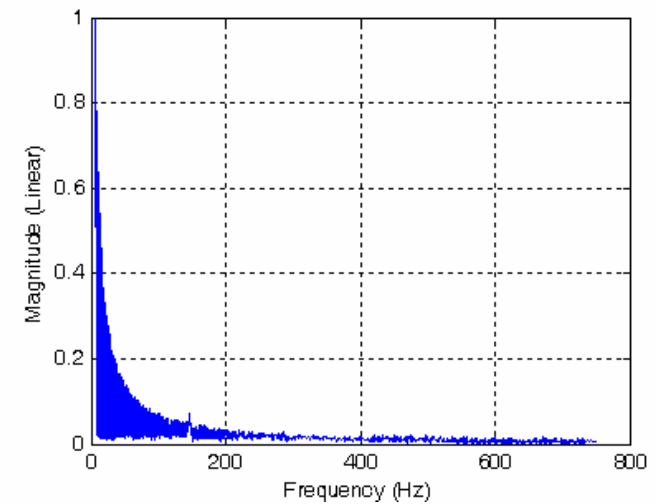

(b)

Figure 8. Switching performance from $200 \mu \mathrm{m}$ beam waist with a capacitor connected in parallel

the magnitude of high frequency components, where the resonant frequencies are. If we reduce the resonant frequency by other methods, we may expect to see the same result. One method is editing a special waveform with the small resonant components by Fourier transfer function. The effect can be neglected if the magnitude of resonant frequency is relatively mall. If the magnitude must be absolute zero to satisfy the requirement, we must cut the resonant frequency range. Loss of some frequency component may suffer Gibbs phenomenon at discontinuous points when we want to edit a special waveform by Fourier transfer function [9].

We edit a special wave function by Matlab ${ }^{\circledR}$ software. Before this we must input a square wave to the switch to get original optical signal. First, we transfer both a square wave and the original optical signal to frequency domain. Find the peak magnitude frequency of the resonant range, similar to Fig. 6(b). In our case, the peak falls on 120, 250 and $300 \mathrm{~Hz}$. Second, reduce the magnitude of square wave frequency spectrum by the range centers on the finding frequencies in first step. It is noted that we reduce the magnitude by the factor with a reciprocal Gaussian distribution. This can avoid magnitude jump, which leads to a Gibbs phenomenon. Finally, do inverse Fourier transform of modulated wave spectrum. A special waveform is generated and is plotted in Fig 9. By reducing the amplitude around the resonant frequency components, the waveform is no longer a square wave. The duty cycle of the edit waveform is $50 \%$ and the repetition rate is 1 Hertz. As we noted, the shape of waveform is highly dependent on the frequency components that are modified. Generally speaking, the rising time of the driving waveform is mainly determined by the high frequency components. This method can be applied to different fabrication batches of MEMS chip to tolerate the manufacturing variations. The Matlab ${ }^{\circledR}$ algorithm code is shown in table I for further reference. There are three major parts in the code, including FFT (Fourier Function Transfer), reduce algorithm and inverse FFT. Both FFT and inverse FFT are standard function provide by the mathematical software. The reduce algorithm is based on the experimental results from the MEMS chip and is user definable.

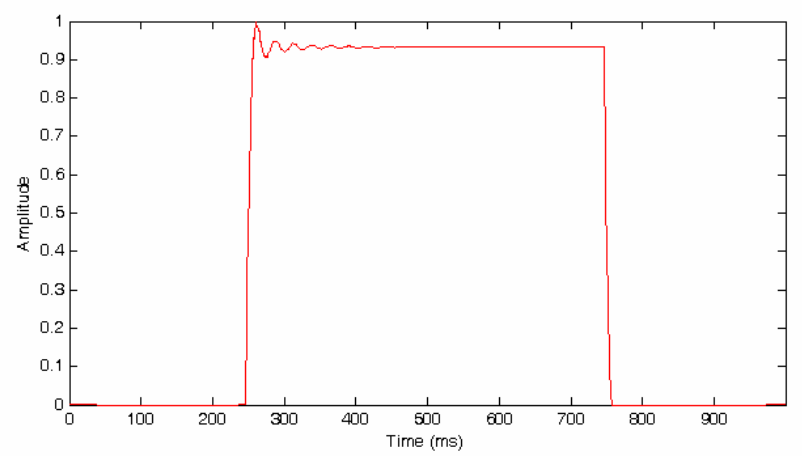

Figure 9. Special driving waveform derived from the code in Table I. 


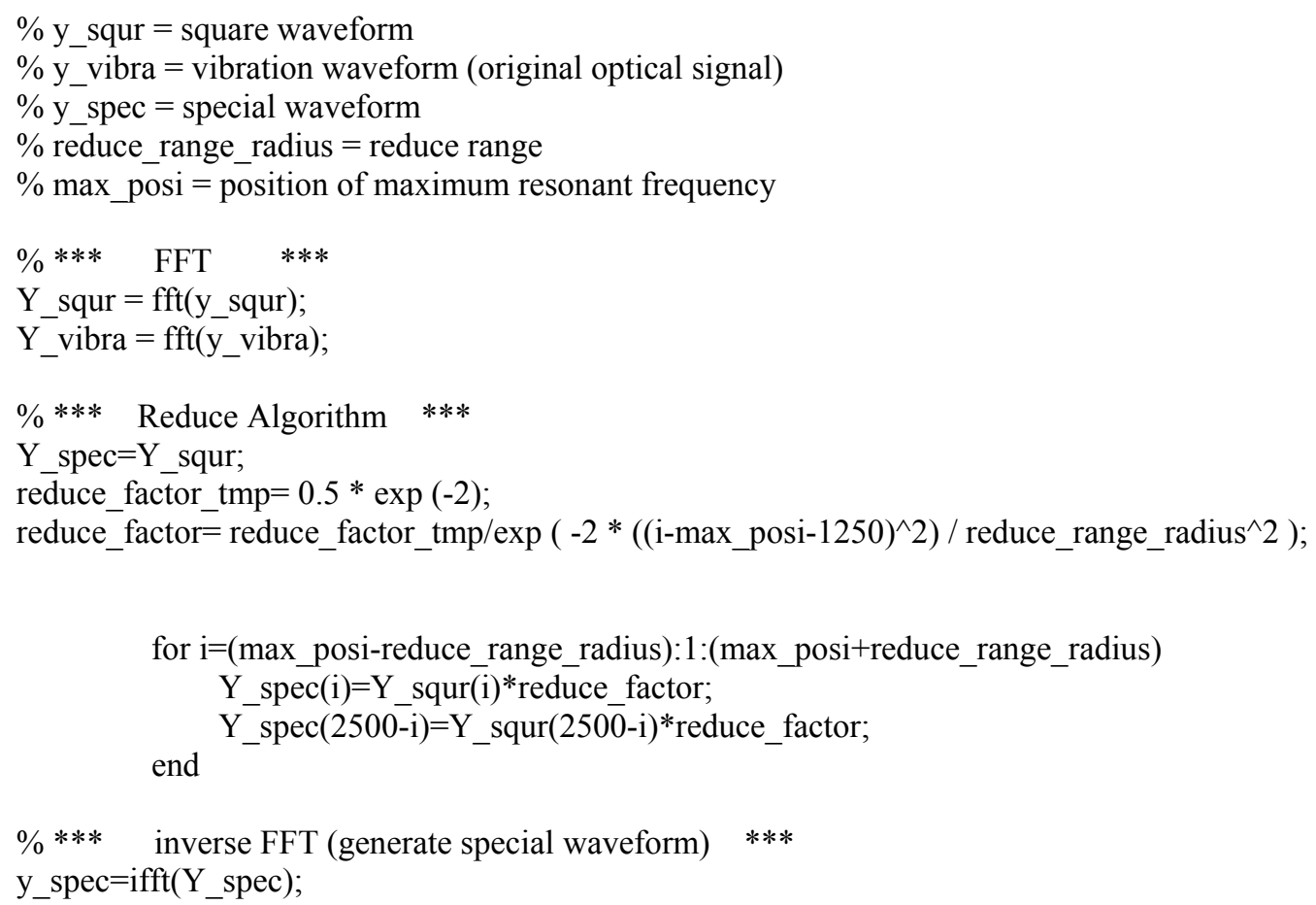

Table I. Part of Matlab code to edit a special waveform

By sending this edited waveform through the function generator, we can see the switching performance in Fig. 10(a). The frequency spectrum of optical signal has small resonant component, as shown in Fig. 10(b). The optical waveform reaches stable state rapidly in about $3 \mathrm{~ms}$ in rising state. Generally speaking, the special waveform can be pre-stored in a memory circuit, such as ROM, and can be released to actuate the MEMS chip upon receiving the trigger signal. The packaged 1 $\times 2$ MEMS optical switch can be integrated with a ROM on the PCB board when it is used in the optical communication network. A detailed switching perform for the on and off states are plotted in Fig. 11 (a) and (b), respectively. The rising time and the falling time the MEMS chip is $3 \mathrm{~ms}$ and $3.1 \mathrm{~ms}$, starting from the electrical driving signal. It can be clearly seen that the special waveform successfully suppressed the vibration of the actuation flap at the resonant frequency.

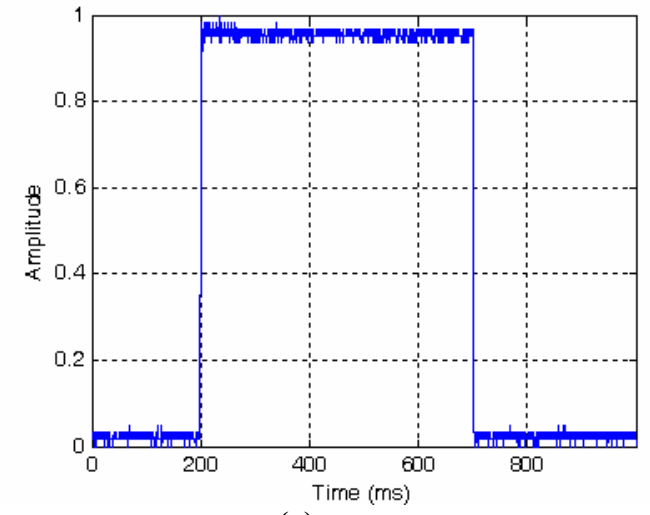

(a)

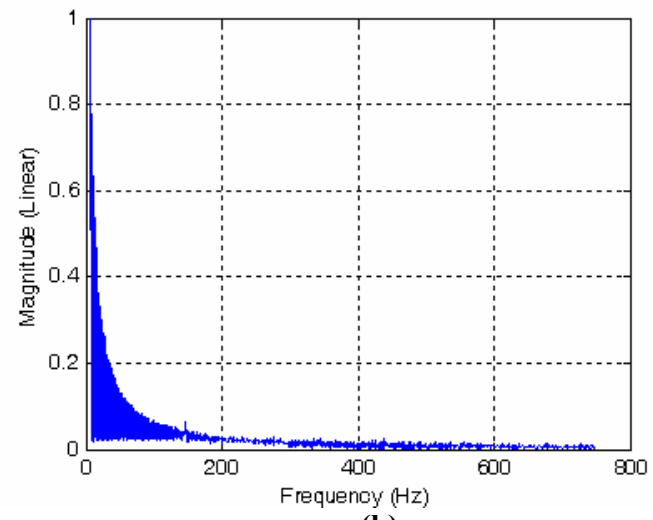

(b)

Figure 10. Switching performance from $62 \mu \mathrm{m}$ beam waist and a special actuation waveform 

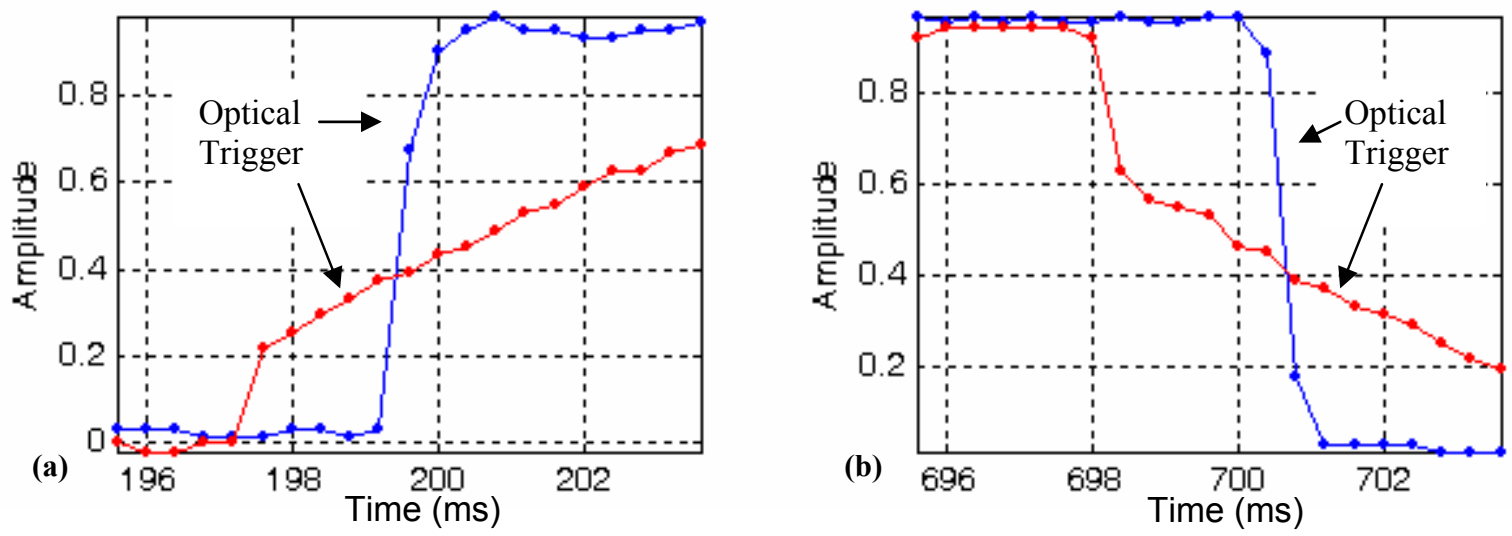

Figure 11. A detailed closed up look of the switching time by using the special waveform

We summarize the progress in table II. Reduce beam size can reduce the switching time because the vibration of the actuation flap can be compensated by large mirror surface area. A capacitor is helpful to filter out undesirable frequency components, which will make the MEMS chip to vibrate less. A special waveform edited according the resonant frequency of the MEMS devices can compensate all improper factors at once. After the modification, we can make the MEMS optical switches work at good performance in a relatively small form factor. Our experimental results show the rising time and fall time of the MEMS switch is $3 \mathrm{~ms}$ and $3.1 \mathrm{~ms}$, respectively. The optical insertion loss caused by the micromirror is $0.45 \mathrm{~dB}$. The overall package size can be reduced to $18 \mathrm{~mm} \times 12 \mathrm{~mm} \times 10 \mathrm{~mm}$.

\begin{tabular}{|l|l|l|}
\hline \multicolumn{1}{|c|}{ performance } & Switching time & Package size \\
\hline $200 \mu \mathrm{m}$ & $\sim 250 \mathrm{~ms}$ & Large, due to collimator and driving circuit \\
\hline $62 \mu \mathrm{m}$ & $\sim 150 \mathrm{~ms}$ & Large, due to driving circuit \\
\hline $62 \mu \mathrm{m}+$ capacitor in parallel & $\sim 5 \mathrm{~ms}$ & Small, only a capacitor \\
\hline $62 \mu \mathrm{m}+$ special waveform & $\sim 3 \mathrm{~ms}$ & Small, waveform in ROM \\
\hline
\end{tabular}

Table II. The switching performance improvement progress.

\section{CONCLUSION}

We design MEMS optical switches with a vertical mirror. The switches are actuated by the electromagnetic force and the actuation voltage is less than 1 volts. The actuation flap is stiction free design and there is no mechanical stopper to absorb kinetic energy of the actuation flap. To improve the switching performance, we re-design the optical beam diameter. By reducing beam size, truncation ratio is increased to accommodate large displacement of the actuation flap at the resonant frequency. After analyzing by Fourier transforms function, only several resonant frequencies are present. A simple low pass filter can reduce unwanted frequencies by connecting a capacitor with the MEMS chip in parallel. No driving circuit or modules are needed. In addition, a special waveform saved in ROM is also an feasible driving method. To get this special waveform, we only need to use the square wave as the driving waveform for the first time, and modify the square wave by minimizing the resonant frequency components. The switching speed by using the special waveform is $3 \mathrm{~ms}$ and $3.1 \mathrm{~ms}$, respectively for rising state and falling state. Low-voltage (typically $0.3 \mathrm{~V}$ ) is achieved to actuate the mirror. The fully packaged switch is small and compact without expensive high-voltage driving circuits. A compact MEMS 1x2 switch with the size of in $18 \times 12 \times 10 \mathrm{~mm}^{3}$ is successfully designed and experimental demonstrated. This work proves that MEMS optical switches are a promising technology for all optical network communication applications. 


\section{REFERENCES}

1. G-D. J. Su et al., "Serpetine Spring Corner Designs for Micro-Electro-Mechanical Systems Optical Switches with Large mirror Mass," Optical review, Vol. 12, No. 4, pp. 339-344, Jul.-Aug. 2005.

2. Franca DR, Blouin A, "All-optical measurement of in-plane and out-of-plane Young's modulus and Poisson's ratio in silicon wafers by means of vibration modes," MEASUREMENT SCIENCE \& TECHNOLOGY, Vol.15, No. 5, pp.859-868, May 2004.

3. Ki Bang Lee, and Liwei Lin "Vertically supported two-directional comb drive," J. Micromech. Microeng., Vol. 15, No.8, pp. 1439-1445, Aug. 2005.

4. Serio B, Hunsinger JJ, Cretin B, "In-plane measurements of microelectromechanical systems vibrations with nanometer resolution using the correlation of synchronous images," REVIEW OF SCIENTIFIC INSTRUMENTS, Vol. 75, No. 10, pp.3335-3341, Part 1, Oct. 2004.

5. Zhou GY, Vj L, Chau FS, Tay FEH, “Micromachined in-plane vibrating diffraction grating laser scanner,"IEEE PHOTONICS TECHNOLOGY LETTERS, Vol. 16, No. 10, pp.2293-2295, Oct. 2004.

6. Chang-Hyeon Ji, Youngjoo Yee, Junghoon Choi, Seong-Hyok Kim, and Jong-Uk Bu, "Electromagnetic $2 \times 2$ MEMS Optical Switch,” Journal of Selected Topics in Quantum Electronics, vol. 10, no. 3, May/June, pp. 545-550, 2004.

7. Lin YC, Chiou JC, Lin WT, Lin YJ, Wu SD, "The design and assembly of surface-micromachined optical switch for optical add/drop multiplexer application," IEEE TRANSACTIONS ON ADVANCED PACKAGING, Vol. 26, No. 3, pp.261-267, Aug. 2003.

8. Guo-Dung John Su, Chen-Wei Chiu, and Fukang Jiang, "Vertical Micromirrors Integrated With Electromagnetic Microactuators for Two-Dimensional Optical Matrix Switches," IEEE PTL, Vol. 17, No. 9, pp.1860-1862, September, 2005.

9. Peter V. O'Neil, “Advanced engineering mathematics $4^{\text {th }}$ ed,” pp.679-681, Wadsworth Publishing Company, 1995. 